\title{
Descrição da Aptidão Inicial para Natação em Lesionados Medulares
}

\author{
Description of Initial Swimming Ability in Patients with \\ Spinal Cord Injury
}

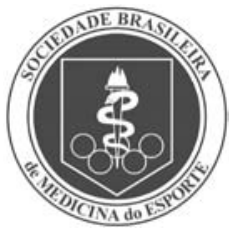

Artigo Original

Ana Cláudia Raposo Melo

Rede Sarah de Hospitais de Reabilitação - Sarah-Centro Brasília - Distrito Federal.

Endereço para correspondência: QSD 43, casa 04 - Taguatinga Sul 72020-430 - DF - Brasil

E-mail: anacraposo@sarah.br, acraposo@correioweb.com.br

Submetido em: 27/08/2008 Versão final recebida em: 15/05/2009 Aceito em: 16/05/2009

\begin{abstract}
RESUMO
Este estudo tem por objetivos verificar o retorno à natação após a lesão medular e descrever a aptidão inicial de lesionados medulares para o nado como parte das atividades do programa de reabilitação no Hospitalar Sarah-Centro. Métodos: Foram avaliados 170 pacientes com diagnóstico de paraplegia e tetraplegia, em três etapas - levantamento de dados em prontuário, entrevista oral e avaliação do padrão do nado. Os resultados apontaram que grande parte dos pacientes não entrou na piscina ou realizou nado após a lesão medular. A avaliação da adaptação ao meio líquido após a lesão medular demonstrou que grande parte dos pacientes realizou a imersão e flutuação de forma independente, porém, apresentando dificuldade em mudar de decúbito dentro da água. Apenas uma pequena porcentagem retomou a natação após a lesão medular; foi também verificada redução no total dos sujeitos que nadavam regularmente antes. A avaliação apontou que os nados crawl e de costas foram realizados por metade dos avaliados; apenas uma pequena parte realizou nado de peito. Conclusão: Após a avaliação dos relatos dos pacientes, verificou-se redução na prática da natação, seja como atividade esportiva, física ou de lazer após a lesão medular, apesar de a aptidão para o nado não ter sofrido grandes alterações.
\end{abstract}

Palavras-chave: natação, lesão medular.

\begin{abstract}
Pysical activity, particularly swimming, that brings a lot of benefits to subjects with spinal cord injury, can be started during rehabilitation. The aims of this study were to describe the initial ability to swim of patients with spinal cord injury during the rehabilitation program at the Sarah Hospital - Center. Methods: 170 patients were evaluated in three steps - medical records data collection, oral interview and swimming evaluation. The results showed that a large proportion of patients did not enter the swimming pool or swam after spinal cord injury. Only a small part returned to swimming after the spinal cord injury. Moreover, reduction in patients who swam regularly before the spinal cord injury was observed. Conclusion. There was a reduction in swimming practice, regardless of being a sport, physical or leisure activity after spinal cord injury.
\end{abstract}

Keywords: swimming, spinal cord injury.

\section{INTRODUÇÃO}

O traumatismo raquimedular acontece por danos nas estruturas medulares, interrompendo a transmissão de impulsos nervosos, alternando negativamente a função motora e sensorial. A lesão medular pode ocorrer por uma lesão traumática, como os acidentes automobilísticos, ou por alguma doença (lesão não traumática), como tumores, infecção, vírus, dentre outras causas ${ }^{(1)}$.

O lesionado medular, como qualquer outra pessoa com deficiência, se beneficia da participação em atividade física, alcançando benefícios, como aumentar a capacidade física(2), auxiliar a independência(3), melhorar o equilíbrio e a destreza na cadeira de rodas ${ }^{(1,4-6)}$, possibilitar a vivência de sensações e movimentos novos ${ }^{(7-9)}$, favorecer o autoconhecimento, a socialização e a readaptação física ${ }^{(4)}$, bem como reduzir os fatores de risco para a saúde, como a obesidade e a hipertensão, promovendo um estilo de vida mais saudável ${ }^{(10)}$.

A atividade física no meio líquido é amplamente indicada para a pessoa com deficiência, pois favorece o desenvolvimento global ${ }^{(11-13)}$. Outro dado a ser ressaltado é que, durante a natação, a movimentação de forma independente é possibilitada, favorecendo a autoestima (2).

A natação para a pessoa com deficiência é considerada por Costa e Duarte $^{(14)}$ como a capacidade do individuo para dominar e deslocar-se de forma independente sobre a água. Concordando com esse conceito, Reis ${ }^{(15)}$, Farias ${ }^{(16)}$ e Velasco ${ }^{(17)}$ definem o ato de nadar como movimentarse na água em diferentes direções, bem como submergir e emergir. Escobar e Burkhardt ${ }^{(18)}$ completam que, para nadar, não há necessidade de preencher os requisitos dos quatro tipos de nado; lembram que nadar é a habilidade de manter-se e locomover-se na água.

A natação beneficia vários sistemas do corpo humano, dentre eles, os sistemas respiratórios ${ }^{(2,9,19,20)}$, sistema cardiovascular ${ }^{(2,8,19,21-23)}$, sistema muscular ${ }^{(2,19)}$, além de benefícios psicossociais ${ }^{(5,23)}$. Essa atividade pode auxiliar o lesionado medular, pois previne doenças do aparelho cardiovascular $(2,8,19,21,22)$, reduz a fraqueza pelo reforço muscular realizado durante os nados, bem como pela resistência oferecida pela água(2,24-27).

Para a pessoa com necessidades especiais, a natação estimula o equilíbrio do tronco e melhora a postura corporal( $2,9,23,24)$, melhora a coordenação motora ${ }^{(11,12,22,24)}$, e favorece a independência funcional(27). O retorno venoso durante o nado é favorecido pela pressão da água(26), bem como pode haver redução da espasticidade pela água quente(22).

As vantagens da natação para paraplégicos e tetraplégicos vão além dos benefícios físicos, dentre elas, a realização de movimentos sem meios auxiliares e a reinserção socia|(11,21). A participação na natação pode auxiliar na prevenção de escaras, por não haver ponto de pressão na pele 
enquanto permanecem na água, e melhorar a autoimagem ${ }^{(4,21,25)}$. Essa atividade aquática pode auxiliar na redução das variações de humor e melhorar a qualidade de vida do lesionado medular ${ }^{(7,19)}$.

A Rede Sarah de Hospitais de Reabilitação acredita nos benefícios da atividade física para lesionados medulares. E oferece, como parte do programa de reabilitação, várias atividades físicas, recreativas e de lazer, dentre elas a natação.

A participação do lesionado medular na atividade aquática-natação no Hospital Sarah-Centro tem por objetivo vivenciar, iniciar e orientar as alterações no padrão de nado, de acordo com a necessidade de cada paciente. A justificativa para realização deste estudo está apoiada no constante relato dos pacientes de não entrarem na piscina, bem como não realizarem o nado, seja como atividade física ou de lazer, após a lesão medular. Outro fator que motivou este estudo foi a ausência na literatura de referência sobre os fatores que possam desmotivar, dificultar ou até impedir a prática da natação em pessoa com lesão medular.

Este trabalho tem por objetivo verificar a prática da natação após a lesão medular, bem como descrever a adaptação ao meio líquido e o padrão de nado dos pacientes ao iniciarem a atividade aquática-natação durante o programa de reabilitação no Hospital Sarah-Centro.

\section{MÉTODOS}

\section{Amostra}

A população deste estudo constituiu-se de 170 pacientes participantes do programa do lesado medular adulto do Hospital Sarah-Centro.

Como critérios de inclusão no estudo, o paciente deveria ter diagnóstico de lesão medular, não apresentar contraindicação à prática da natação, ter idade mínima de 18 anos para fornecer por escrito o consentimento livre e esclarecido, seguindo assim as orientações da Declaração de Helsinque e as normas da Resolução 196/96 do Conselho Nacional de Saúde. Após ser orientados sobre a proposta deste estudo, os pacientes foram esclarecidos de que a avaliação realizada é comum a todos os com lesão medular que participam da natação.

\section{Procedimentos}

Este estudo foi realizado em três etapas. Primeiramente foi realizado um levantamento no prontuário eletrônico de cada paciente que iniciaria a natação, colhendo dados como: idade, sexo, classificação da lesão medular e tempo de lesão. Para a classificação da lesão medular foram utilizadas apenas a paraplegia e a tetraplegia, sem diferenciação do nível da lesão, por ser esta classificação suficiente para avaliação e orientação ao nado.

Em uma segunda etapa, os pacientes participantes deste estudo foram então entrevistados por um professor de educação física. Nesse momento foi questionado o histórico de atividade física, da atividade aquática e padrão de nado antes e após a lesão medular, sendo considerado nado a forma de realização do deslocamento na água.
Por último, foram analisadas a imersão, flutuação, mudança de decúbito e deslocamento na água e a avaliação do padrão atual de nado. A avaliação do nado inclui itens como braçada, respiração e nado completo durante um percurso de 10 metros.

A coleta de dados em todas as etapas deste estudo foi realizada por um profissional de educação física, que atua no setor de hidroterapia do Hospital Sarah-Centro, com a atividade aquática-natação.

\section{Caracterização do estudo}

Este estudo é de caráter descritivo, tendo por objetivo informar a aptidão de pacientes com lesão medular ao iniciar a natação durante programa de reabilitação. Para a análise estatística, os dados coletados na entrevista originaram um banco de dados e foram analisados através da média e percentual.

\section{RESULTADOS}

A amostra do estudo foi de 170 pacientes, sendo 56 do sexo feminino (32,9\%) e 114 do masculino (67,1\%). Verificou-se no momento do estudo que a idade média dos sujeitos era de 30,7 anos. A classificação da lesão medular revelou 109 pacientes com paraplegia (64,2\%) e 61 com tetraplegia (35,8\%).

Os pacientes apresentaram, ao iniciar a atividade aquática-natação, a média de tempo de ocorrência da lesão medular, coletada em prontuário, de 2,5 anos; o menor tempo de lesão medular era de três meses e o maior, de 17 anos.

O histórico de atividade física antes da lesão medular, avaliado através do relato dos pacientes, demonstrou que 85 deles participavam esporadicamente de atividades físicas (49,9\%) e 43 eram sedentários (24,7\%). A participação regular em atividade física foi relatada por 45 pacientes (25,7\%). Após a lesão medular a grande maioria dos pacientes $(98,6 \%)$ não participou de atividade física; apenas três $(1,4 \%)$ informaram participar regularmente de atividade física, conforme demonstrado na tabela 1

Na avaliação da participação em natação a maioria (90\% - 51,5\% paraplégicos e 28,5\% tetraplégicos) informou que antes da lesão medular nadava em piscina, rios, lagos, mar, dentre outros locais, sendo realizada como atividade física regular ou de lazer, mesmo que esporadicamente. Após a lesão medular 158 pacientes informaram que não nadaram. Outro dado interessante observado foi que apenas uma minoria $(7,2 \%)$ dos pacientes retomou a natação, mesmo como atividade para o lazer ou realizada esporadicamente. Esses dados estão apresentados na tabela 2.

O padrão de nado antes da lesão medular informado por 44,1\% dos pacientes era de crawl, com diferentes padrões de respiração. Os nados crawl e de costas eram executados por 11,6\% dos pacientes. O nado submerso ou apenas o mergulho em pequenas distâncias era executado por 14,3\% dos pacientes e 18,5\% informaram que não sabiam nadar antes da lesão medular. Esses dados podem ser mais bem avaliados na tabela 3.

Tabela 1. Avaliação do histórico em atividade física.

\begin{tabular}{|c|c|c|c|c|c|c|c|c|c|c|c|c|c|}
\hline \multirow{4}{*}{ Atividade física } & \multicolumn{8}{|c|}{ Antes da lesão medular } & \multicolumn{5}{|c|}{ Após a lesão medular } \\
\hline & \multicolumn{4}{|c|}{ sexo } & \multicolumn{4}{|c|}{ Nível LM } & \multicolumn{2}{|c|}{ sexo } & \multicolumn{3}{|c|}{ Nível LM } \\
\hline & \multicolumn{2}{|c|}{ feminino } & \multicolumn{2}{|c|}{ masculino } & \multicolumn{2}{|c|}{ paraplegia } & \multicolumn{2}{|c|}{ tetraplegia } & feminino & masculino & paraplegia & \multicolumn{2}{|c|}{ tetraplegia } \\
\hline & Freq & med & Freq & med & Freq & med & Freq & med & Freq. med & Freq med & Freq med & Freq & med \\
\hline Sedentário & 24 & $14,3 \%$ & 17 & $10 \%$ & 33 & $18 \%$ & 9 & $5,7 \%$ & $32,9 \%$ & $114 \quad 67,1 \%$ & $67,1 \%$ & 56 & $33,9 \%$ \\
\hline Esporádico & 24 & $14,3 \%$ & 60 & $35,7 \%$ & 49 & $29,1 \%$ & 36 & $20,1 \%$ & & & & & \\
\hline Regular & 8 & $4,3 \%$ & 37 & $21,4 \%$ & 32 & $18,5 \%$ & 12 & $7,1 \%$ & & & & & \\
\hline Total & 56 & $32,9 \%$ & 114 & $67,1 \%$ & 114 & $67,1 \%$ & 56 & $32,9 \%$ & $32,9 \%$ & $114 \quad 67,1 \%$ & $67,1 \%$ & 56 & $33,9 \%$ \\
\hline
\end{tabular}


Tabela 2. Avaliação da atividade aquática-nado.

\begin{tabular}{|c|c|c|c|c|c|c|c|c|c|c|c|c|c|c|c|c|}
\hline \multirow{4}{*}{ nado } & \multicolumn{8}{|c|}{ Antes da lesão medular } & \multicolumn{8}{|c|}{ Após a lesão medular } \\
\hline & \multicolumn{4}{|c|}{ sexo } & \multicolumn{4}{|c|}{ Nível LM } & \multicolumn{4}{|c|}{ sexo } & \multicolumn{4}{|c|}{ Nível LM } \\
\hline & \multirow{2}{*}{\multicolumn{2}{|c|}{$\begin{array}{l}\text { feminino } \\
\text { Freq med }\end{array}$}} & \multicolumn{2}{|c|}{ masculino } & \multicolumn{2}{|c|}{ parap } & \multicolumn{2}{|c|}{ tetrap } & \multicolumn{2}{|c|}{ feminino } & \multicolumn{2}{|c|}{ masculino } & \multicolumn{2}{|c|}{ parap } & \multicolumn{2}{|c|}{ tetrap } \\
\hline & & & Frec & med & Freq & med & Freq & med & Freq & med & Freq & med & Freq & med & Freq & med \\
\hline $\operatorname{sim}$ & 32 & $18,5 \%$ & 104 & $61,5 \%$ & 87 & $51,6 \%$ & 49 & $28,5 \%$ & 9 & $5,7 \%$ & 2 & $1,4 \%$ & 9 & 5,7 & 2 & $1,4 \%$ \\
\hline não & 24 & $14,27 \%$ & 9 & $5,8 \%$ & 24 & $14,2 \%$ & 9 & $5,8 \%$ & 48 & $28,5 \%$ & 111 & $65,7 \%$ & 103 & $60 \%$ & 56 & $32,8 \%$ \\
\hline Total & 56 & $32,7 \%$ & 114 & $67,3 \%$ & 112 & $65,8 \%$ & 58 & $34,2 \%$ & 57 & $34,2 \%$ & 113 & $67,1 \%$ & 112 & 65,7 & 58 & 34,3 \\
\hline
\end{tabular}

Tabela 3. Padrão de nado antes da lesão medular.

\begin{tabular}{|c|c|c|c|c|c|c|c|c|}
\hline \multirow{3}{*}{ nado } & \multicolumn{4}{|c|}{ sexo } & \multicolumn{4}{|c|}{ Nível LM } \\
\hline & \multicolumn{2}{|c|}{ feminino } & \multicolumn{2}{|c|}{ masculino } & \multicolumn{2}{|c|}{ parap } & \multicolumn{2}{|c|}{ tetrap } \\
\hline & Freq & med & Freq & med & Freq & med & Freq & med \\
\hline Não nadava & & $12, \%$ & 9 & $5,7 \%$ & 22 & $12,8 \%$ & 9 & $5,7 \%$ \\
\hline Submerso & 9 & $5,7 \%$ & 14 & $7,5 \%$ & 17 & $10 \%$ & 7 & $4,3 \%$ \\
\hline Utilitário & 2 & $1,5 \%$ & 9 & $5,7 \%$ & 9 & $5,7 \%$ & 2 & $1,5 \%$ \\
\hline Utilitário/submerso & 9 & $5,7 \%$ & 22 & $12 \%$ & 22 & $12 \%$ & 9 & $5,7 \%$ \\
\hline $\begin{array}{l}\text { Crawl cabeça fora } \\
\text { da água }\end{array}$ & 2 & $1,5 \%$ & 14 & $8,5 \%$ & 7 & $4,3 \%$ & 9 & $5,7 \%$ \\
\hline Crawl resp. frontal & 4 & $2,8 \%$ & 2 & $1,5 \%$ & 7 & $4,3 \%$ & & \\
\hline Crawl completo & 2 & $1,5 \%$ & 9 & $5,7 \%$ & 7 & $4,3 \%$ & 4 & $2,8 \%$ \\
\hline $\begin{array}{l}\text { Crawl costas } \\
\text { completo }\end{array}$ & 2 & $1,5 \%$ & 17 & $10 \%$ & 12 & $7,1 \%$ & 7 & $4,3 \%$ \\
\hline Crawl costas peito & & & 22 & $12 \%$ & 12 & $7,1 \%$ & 7 & $4,3 \%$ \\
\hline Total & 52 & $32,2 \%$ & 118 & $67,8 \%$ & 115 & $65,6 \%$ & 55 & 34,3 \\
\hline
\end{tabular}

A independência no meio líquido foi avaliada em quesitos como imersão e mudança de decúbito. Apontou que a imersão é realizada pela maioria dos pacientes $(94,2 \%$ ) e apenas $5,8 \%$ não a fizeram, mesmo que apenas do rosto. A imersão testada foi realizada de diferentes formas, conforme pode ser verificado na tabela 4 . Vale ressaltar que os pacientes que não realizaram a imersão, mesmo com apoio do professor ou da borda, não nadavam antes da lesão medular. A mudança de decúbito (ventral/dorsal e dorsal/ventral) foi realizada com auxilio por 62,9\% dos pacientes. Um dado interessante observado foi que a mudança de decúbito realizada de forma independente $(31,4 \%)$ foi executada por pacientes paraplégicos e que nadavam antes da lesão medular. Os dados sobre a imersão e a mudança de decúbito estão apresentados na tabela 4.

A flutuação em ventral foi realizada em diferentes formas pela maioria dos pacientes $(98,5 \%)$, conforme se pode verificar na tabela 5 . Todos os pacientes que realizaram a flutuação em ventral de forma independente (109) nadavam antes da lesão medular. Já a avaliação da flutuação dorsal demonstrou que 97,2\% dos pacientes conseguiram flutuar de costas, seja de forma independente, com apoio do professor ou com a utilização do flutuador. A flutuação dorsal de forma independente foi mais evidente nos homens.

Tabela 4. Avaliação da independência em meio líquido.

\begin{tabular}{|c|c|c|c|c|c|c|c|c|c|c|c|c|c|c|c|c|}
\hline \multicolumn{9}{|c|}{ imersão } & \multicolumn{8}{|c|}{ Mudança decúbito } \\
\hline & \multirow{2}{*}{\multicolumn{2}{|c|}{$\begin{array}{l}\text { feminino } \\
\text { Freq med }\end{array}$}} & \multirow{2}{*}{\multicolumn{2}{|c|}{$\begin{array}{l}\text { masculino } \\
\text { Freq med }\end{array}$}} & \multirow{2}{*}{\multicolumn{2}{|c|}{\begin{tabular}{c}
\multicolumn{2}{c}{ parap } \\
Freq med
\end{tabular}}} & \multicolumn{2}{|c|}{ tetrap } & \multicolumn{2}{|c|}{ feminino } & \multicolumn{2}{|c|}{ masculino } & \multicolumn{2}{|c|}{ parap } & \multicolumn{2}{|c|}{ tetrap } \\
\hline & & & & & & & Fred & med & Freq & med & Freq & med & Freq & med & Freq & med \\
\hline Não realiza & 7 & $4,3 \%$ & 2 & $1,5 \%$ & 9 & $5,7 \%$ & & & 9 & $5,7 \%$ & & & 7 & $4,2 \%$ & 2 & $1,5 \%$ \\
\hline Apoio professor & 12 & $7,1 \%$ & 14 & $8,5 \%$ & 7 & $4,3 \%$ & 20 & $11,5 \%$ & & $24,2 \%$ & 66 & $38,7 \%$ & 64 & $37,1 \%$ & 44 & $25,8 \%$ \\
\hline Independente & 22 & $12,5 \%$ & 95 & $55,7 \%$ & 83 & $48,5 \%$ & 35 & $20 \%$ & 4 & $2,8 \%$ & 49 & $28,6 \%$ & 39 & $22,9 \%$ & 14 & $8,5 \%$ \\
\hline Total & 53 & $31 \%$ & 123 & $72,9 \%$ & 108 & $64,2 \%$ & 62 & $35,8 \%$ & 55 & $32,7 \%$ & 115 & $67,3 \%$ & 110 & 64,2 & 60 & 35,8 \\
\hline
\end{tabular}

Tabela 5. Avaliação da flutuação ventral e dorsal.

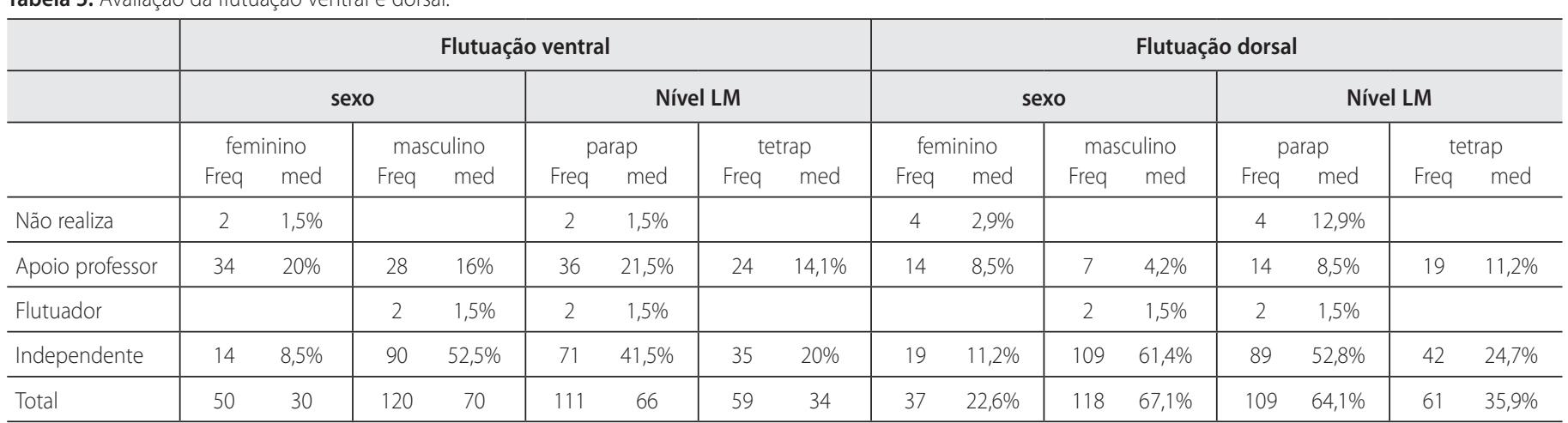


Quanto à avaliação do padrão de nado após a lesão medular, verificou-se que apenas 2,9\% dos pacientes executaram os quatro nados. 0 nado de costas foi o mais facilmente executado neste estudo, tanto por paraplégicos quanto por tetraplégicos, que nadavam ou não antes da lesão medular. O nado de costas com remada é executado por 75,6\% dos pacientes (47,1\% paraplégicos e 28,5\% tetraplégicos). Apenas uma minoria (5,7\%) não conseguiu nadar de costas (Tabela 6).

Tabela 6. Padrão de nado após a lesão medular - nado de costas.

\begin{tabular}{l|cc|cc|cc|cc}
\hline \multirow{2}{*}{ nado } & \multicolumn{4}{c|}{ sexo } & \multicolumn{3}{c}{ Nível LM } \\
\hline & \multicolumn{2}{c|}{$\begin{array}{c}\text { feminino } \\
\text { Freq }\end{array}$} & \multicolumn{2}{c|}{ med } & masculino & \multicolumn{2}{c}{ parap } & \multicolumn{2}{c}{ tetrap } \\
Freq & med & Freq med & Freq med \\
\hline Não realiza & 9 & $5,7 \%$ & 2 & $1,5 \%$ & 9 & $5,7 \%$ & 2 & $1,5 \%$ \\
\hline Braçada alternada & 24 & $14,2 \%$ & 14 & $8,5 \%$ & 9 & $5,7 \%$ & 4 & $2,8 \%$ \\
\hline Braçada dupla & & & 2 & $1,5 \%$ & 9 & $1,5 \%$ & & \\
\hline remada. & 24 & $14,2 \%$ & 86 & $48,7 \%$ & 80 & $47,1 \%$ & 49 & $29,5 \%$ \\
\hline completo & & & 9 & $5,7 \%$ & 4 & $2,8 \%$ & 4 & $2,8 \%$ \\
\hline Total & 57 & $34,1 \%$ & 113 & $65,9 \%$ & 111 & $63,4 \%$ & 59 & $36,6 \%$ \\
\hline
\end{tabular}

Quanto ao nado crawl, verificou-se que 41,3\% dos pacientes não conseguiram executá-lo, mesmo os que nadavam antes da lesão medular. O nado crawl com diferentes formas de respiração é realizado em maior número por paraplégicos (Tabela 7).

Tabela 7. Padrão de nado após a lesão medular.

\begin{tabular}{l|cc|cc|cc|cc}
\hline \multicolumn{1}{c|}{ sexo } & \multicolumn{4}{c}{ Nível LM } \\
\hline \multicolumn{1}{c|}{ nado } & \multicolumn{9}{c|}{ feminino } & \multicolumn{2}{c|}{ masculino } & \multicolumn{2}{c}{ parap } & \multicolumn{2}{c}{$\begin{array}{c}\text { tetrap } \\
\text { med }\end{array}$} \\
\hline Freq & med & Freq & med & Freq & med & Freq \\
\hline Não realiza & 39 & $22,8 \%$ & 31 & $18,5 \%$ & 36 & $20,3 \%$ & 36 & $21,1 \%$ \\
\hline Braçada resp. lateral & 8 & $4,3 \%$ & 39 & $22,8 \%$ & 34 & $20 \%$ & 12 & $7,2 \%$ \\
\hline Braçada resp. frontal & 8 & $4,3 \%$ & 20 & $11,5 \%$ & 24 & $12,8 \%$ & 4 & $2,8 \%$ \\
\hline Braçada sem resp. & 4 & $2,8 \%$ & 9 & $5,7 \%$ & 7 & $4,2 \%$ & 4 & $2,8 \%$ \\
\hline completo & & & 12 & $7,2 \%$ & 9 & $5,7 \%$ & 4 & $2,8 \%$ \\
\hline Total & 59 & $34,2 \%$ & 111 & $65,8 \%$ & 110 & $63 \%$ & 60 & $37 \%$ \\
\hline
\end{tabular}

Em relação ao nado de peito, foi evidenciado que este é executado em menor frequência $(49,9 \%)$ pelos pacientes avaliados. Vale ressaltar que todos os que executaram o nado de peito sabiam nadar antes da lesão medular (Tabela 8).

Quanto ao nado borboleta, a grande maioria dos pacientes não o realiza (97,1\%). Os dados coletados sobre o padrão de nado após a lesão medular e suas variações são apresentados de forma mais detalhada na tabela 6 (Tabela 9).

\section{DISCUSSÃO}

A classificação da lesão medular - paraplegia e tetraplegia - adotada neste estudo concorda com Cowart(28) e Albright ${ }^{(29)}$, ao apontarem que a classificação da lesão medular pode auxiliar o profissional a identificar a aptidão inicial para o nado, bem como identificar e corrigir erros, visando melhora do padrão inicial.
Tabela 8. Padrão de nado após a lesão medular - nado de peito.

\begin{tabular}{|c|c|c|c|c|c|c|c|c|}
\hline \multicolumn{9}{|c|}{ peito } \\
\hline \multirow[b]{2}{*}{ nado } & \multicolumn{4}{|c|}{ sexo } & \multicolumn{4}{|c|}{ Nível LM } \\
\hline & $\begin{array}{l}\text { fen } \\
\text { Freq }\end{array}$ & $\begin{array}{l}\text { nino } \\
\text { med }\end{array}$ & $\begin{array}{l}\text { mas } \\
\text { Freq }\end{array}$ & $\begin{array}{l}\text { culino } \\
\text { med }\end{array}$ & $\begin{array}{r}p \\
\text { Freq }\end{array}$ & $\begin{array}{l}\text { arap } \\
\text { med }\end{array}$ & $\begin{array}{r}\text { te } \\
\text { Freq }\end{array}$ & $\begin{array}{l}\text { rap } \\
\text { med }\end{array}$ \\
\hline Não realiza & 44 & $25,7 \%$ & 42 & $25 \%$ & 56 & $32,8 \%$ & 29 & $17,1 \%$ \\
\hline \multicolumn{9}{|l|}{ Braçada resp. lateral } \\
\hline Braçada resp. frontal & 2 & $1,5 \%$ & 2 & $1,5 \%$ & 2 & $1,5 \%$ & 2 & $1,5 \%$ \\
\hline Braçada resp. bra. & 7 & $4,2 \%$ & 73 & $42,1 \%$ & 53 & $31,4 \%$ & 28 & $15,7 \%$ \\
\hline Total & 53 & $31,4 \%$ & 117 & $68,9 \%$ & 112 & $65,7 \%$ & 59 & $34,3 \%$ \\
\hline
\end{tabular}

Tabela 9. Padrão de nado após a lesão medular - nado borboleta.

\begin{tabular}{l|cc|cc|cc|cc}
\hline \multicolumn{1}{c|}{ borboleta } & \multicolumn{3}{c}{ Nível LM } \\
\hline \multirow{2}{*}{ nado } & \multicolumn{3}{c|}{ feminino } & \multicolumn{2}{c|}{ masculino } & \multicolumn{2}{c}{ parap } & \multicolumn{2}{c}{$\begin{array}{c}\text { tetrap } \\
\text { med }\end{array}$} \\
\hline Não realiza & 56 & $32,6 \%$ & 112 & $64,6 \%$ & 105 & $61,4 \%$ & 61 & $35,8 \%$ \\
\hline Braçada resp. lateral & & & 2 & $2,8 \%$ & 4 & $2,8 \%$ & & \\
\hline Total & & & 170 & $100 \%$ & 170 & $100 \%$ & 170 & $100 \%$ \\
\hline
\end{tabular}

Almeida e Tonello(13) apontam que os lesados medulares conhecem e concordam com os benefícios, tanto físicos como sociais, da participação na natação, porém, na prática, os dados encontrados neste estudo demonstraram ampla redução $(84,3 \%)$ na natação após a lesão medular. Inclusive, pode-se observar redução de 1,4\% na participação em pacientes que nadavam regularmente antes da lesão medular.

Outro dado importante foi que $17,7 \%$ dos pacientes informaram que entraram na piscina após a lesão medular para realizar exercícios de hidroterapia. O baixo retorno à natação, seja como atividade física regular, esporte ou lazer, pode ser justificado por vários fatores, como a dificuldade de acesso à piscina ou mesmo pela baixa participação regular em atividade física antes da lesão medular, porém, essas informações não foram colhidas, sendo necessária uma avaliação adequada dessas questões em outro estudo.

O retorno à natação, segundo Innenmoser ${ }^{(30)}$, pode ser dificultado pelas modificações corporais ou redução das capacidades orgânicas após uma lesão medular. A presença de alterações corporais após a lesão medular, associada ao receio do fracasso ou por acreditar que não conseguiria nadar devido à ausência de movimentos em membros inferiores, ter influenciado no baixo percentual de retorno à natação encontrados neste estudo.

Segundo Albright ${ }^{(29)}$, é importante identificar elementos que auxiliem na segurança do lesionado medular em atividades no meio líquido, como a flutuação e mudança de decúbito. A avaliação da adaptação ao meio líquido após a lesão medular identificou neste estudo a dificuldade na flutuação e mudança de decúbito de forma independente, porém, essas atividades foram realizadas com apoio do professor como forma de orientação para continuidade fora do ambiente hospitalar.

A dificuldade em realizar a flutuação ou a mudança de decúbito de forma independente pode ter sido influenciada por ausência ou redução de movimento decorrentes da lesão medular, pela presença de espasticidade, pela não adaptação às mudanças na flutuação e/ou outros fatores que necessitam de avaliação mais criteriosa. 
A variação no padrão inicial de nado encontrada neste estudo concorda com Albright ${ }^{(29)}$, quando esse autor destaca que o padrão de nado após a lesão medular deverá ser funcional, de acordo com a altura da lesão medular. Isso pode explicar que pacientes, que não realizavam o nado de peito antes da lesão medular, em apenas uma aula se adaptaram a esse nado.

O aumento no nado de peito (braçada com respiração) pode ser explicado pela provável melhora da estabilidade do tronco durante a braçada, bem como com a semelhança com a braçada do nado submerso, que grande parte dos pacientes realizava antes da lesão medular.

Ainda concordando com Albright ${ }^{(29)}$ quanto à funcionalidade do nado, este estudo demonstrou aumento no número de pacientes que realizam o nado de costas após a lesão medular, fato este possivelmente relacionado com a impossibilidade de executar o nado em posição ventral; outros fatores podem ter influenciado na dificuldade em realizar o nado em decúbito ventral.

A diminuição do nado utilitário (cachorrinho) e do nado crawl com cabeça fora da água pode ter ocorrido devido à redução ou ausência dos movimentos de membros inferiores, importantes para esses nados. Outro fator que contribui para a redução do nado utilitário foi a orientação de cuidados para que os pés não arrastem no fundo da piscina, uma vez que nadar com a cabeça dentro da água minimiza essa possibilidade.

Aos pacientes que não realizaram o nado de forma independente foi possibilitada, mesmo com auxílio da borda ou do professor, a execução de elementos com flutuação e adaptação. O auxílio do professor, que passa a segurança necessária para melhor execução dos elementos propostos, pode minimizar a possibilidade de sensações desagradáveis durante a atividade aquática. A participação na natação, mesmo que apenas em um dia de orientação, pode significar uma etapa inicial para a realização do nado independente fora do ambiente hospitalar.

As limitações apresentadas neste estudo incluem a impossibilidade de confronto entre a informação dos pacientes quanto ao histórico sobre a atividade física e o nado, pois não há referência à participação em atividade física em prontuário. Uma forma de confrontar essas informações seria checar junto ao clube, academia ou outro local onde o paciente realiza esta atividade; porém, parte dos pacientes informou que a atividade física era realizada apenas esporadicamente ou, ainda, ao ar livre.

\section{REFERÊNCIAS BIBLIOGRÁFICAS}

1. Rede SARAH de Hospitais de Reabilitação. Lesão Medular. Capturado no dia 10 de novembro de 2008. Disponível em http://www.sarah.br/paginas/doenças/po/p_08_lesao_medular. htm\#Anchor-49708 2008

2. Massaud MG, Correa CR. Natação para adultos. Rio de janeiro: Sprint, 2001.

3. Collen FM, Wade DT, Robb GF, Bradshan CM. The Rivermead Mobility Index: a further development of the Rivermead Motor Assessment. Int Disabil Stud. 1991;13(2):50-4.

4. Souza PA. O esporte na paraplegia e tetraplegia. Rio de Janeiro: Guanabara Koogan, 1994.

5. Rosadas SC. Educação física para deficientes. Rio de Janeiro: Ateneu, 1986.

6. Schutz LK. The wheelchair athlete. In: Buschbacher RM, Braddom RL, editors. Sports medicine and rehabilitation: a sport-specific approach. Philadelphia: Hanley \& Belfus, Inc, 1984;267-74

7. Jackson RW. Sport for the spinal paralyzed person. Paraplegia. 1987;25:301-4.

8. Santiago MC, Coyle CP, Kinney WB. Aerobic exercise effect on individuals with physical disabilities. Arch Phys Med Rehabil. 1993;74(11):1192-8..

9. Campion MR. Adult hydrotherapy: a practical approach. Oxford: Heinemann Medical Books, 1990 .

10. Rimmer JH. Healthy promotion for people with disabilities: The emerging paradigm shift from disability prevention to prevention of secondary conditions. Phys Ther. 1999;79 495-502.

11. Freudenhein AM. O nadar: uma habilidade motora revisitada. São Paulo: Edusp, 1995.

12. Chachan AM. Reliability and validity of the aquatic adjustment test for children with disabilities. 2001. Anais do 13rd International Symposium Adapted Physical Activity, Viena.

13. Almeida PA, Tonello MG. Benefícios da natação para alunos com lesão medular. 2007. Capturado no dia 10 de fevereiro de 2008. Disponível em http://www.efdeportes.com Revista Digital - Buenos Aires - Año 11 - No 106 - Marzo de 2007.

14. Costa AM, Duarte E. Aspectos teóricos da atividade aquática para portadores de deficiência. In Educação Física e Esporte para Deficientes - coletânea. Organizadora Freitas, OS. Ministério do Esporte, Uberlândia, 2002.
A relevância deste estudo foi confirmar que a grande maioria dos pacientes, após a lesão medular, não retomou à natação, seja como atividade física regular ou como lazer. A confirmação desse fato se torna importante, pois durante o programa de reabilitação essa atividade pode ser indicada e orientada, para que o paciente inicie a natação em ambiente hospitalar e possa dar continuidade ao término desse período.

\section{CONSIDERAÇÕES FINAIS}

Este estudo foi apenas de caráter descritivo do ponto de partida para a natação em pacientes internados em programa de reabilitação no Hospital Sarah-Centro. Outros estudos são necessários para avaliar a influência do nível de lesão medular nas alterações do padrão de nado e os motivos que impediram a continuidade da natação, entre outras questões relacionadas.

Este estudo evidencia uma drástica redução da participação na natação após uma lesão medular, seja como atividade física regular ou como lazer.

A identificação na redução, após a lesão medular, na prática de atividade física ou, em específico, da natação, tem um papel importante na tentativa de reverter esse fato, pois o lesionado medular, assim como todas as pessoas, deve participar de uma atividade física regular.

Os dados obtidos neste estudo demonstram redução na prática da natação após a lesão medular. O padrão de nado executado após a lesão medular evidenciou a possibilidade de grande parte dos pacientes praticar natação.

Outro dado importante a ser avaliado em estudos futuros será o quanto o tempo de lesão pode influenciar na participação da atividade aquática-natação, pois, quanto maior o período, maior a adaptação às alterações provocadas pela lesão medular. Seria importante avaliar em estudos futuros quais os motivos que dificultaram ou impediram o retorno à natação ou a outra atividade física, esportiva ou de lazer após a lesão medular.

A participação na natação durante o período de internação, mesmo com apenas um dia de orientação, pode possibilitar o retorno a essa prática, seja como atividade física, esportiva ou de lazer.

O autor declara não haver qualquer potencial conflito de interesses referente a este artigo.
15. Reis JW. A natação na sua expressão psicomotriz. Porto Alegre Editora da Universidade do Rio Grande do Sul, 1982.

16. Farias SF. Natação: ensine a nadar. Florianópolis: Editora da UFSC, 1994.

17. Velasco CG. Natação segundo a psicomotricidade. Rio de Janeiro: Sprint, 1994.

18. Escobar MO, Burkhardt, R. Natação para portadores de deficiência. Rio de Janeiro: Ao Livro Técnico, 1985.

19. Shepard RJ. Benefits of sport and physical activity for the disabled: implications for the individual and for society. Scand J Rehab Med. 1991;23:51-9.

20. Washburn RA, Figoni SF. Physical activity and chronic cardiovascular disease prevention in spinal cord injury: a comprehensive literature review. Top Spinal Cord Inj Rehabil. 1999;3:16-32.

21. Burkhardt R, Escobar MO. Natação para portadores de deficiências. Rio de Janeiro: Ao Livro Técnico, 1985. 22. Bromley I. Paraplegia \& Tetraplegia - Um guia teórico-prático para fisioterapeutas, cuidadores e familiares. $4^{\text {a }}$ ed. Rio de Janeiro: Revinter, 1997.

23. Campion MR. Hidroterapia. Princípios e prática. São Paulo: Manole, 2000.

24. PaeslackV. Esporte para os paraplégicos. In: Hullemann KD, editor. Medicina esportiva: clínica e prática. São Paulo: EPU/Educação Física da Universidade de São Paulo, 1978.

25. Méier M. Atividade física para deficiente. Ministério da Educação e Cultura. Secretaria de Educação Física e Desportos. Brasília: SEED/MEC, 1981.

26. Skinner AT, Thomson AM. Duffield: Exercícios na água. 3a ed. São Paulo: Manole, 1985.

27. Chatard JC, Lavoie JM, Ottoz H, Randaxhe P, Cazorla G, Lacour JR. Physiological aspects of swimming performance for persons with disabilities. Med Sci Sports Exerc. 1992;24:1276-82,

28. Cowart J. Teaching swim skills to the hard to reach students. Palaestra, 1998; 32-8.

29. Albright C. Swimming techniques for individuals with physical disabilities. Palaestra, 1995;11(2),16-21.

30. Innenmoser J. Natação para deficientes. Natação para principiantes. Lisboa: Editora Casa do Livro, 1979. 